\title{
Bosonization and the eikonal expansion: similarities and differences
}

\author{
Peter Kopietz \\ Institut für Theoretische Physik der Universität Göttingen, \\ Bunsenstr.9, D-37073 Göttingen, Germany
}

(January 23, 1996)

\begin{abstract}
We compare two non-perturbative techniques for calculating the singleparticle Green's function of interacting Fermi systems with dominant forward scattering: our recently developed functional integral approach to bosonization in arbitrary dimensions, and the eikonal expansion. In both methods the Green's function is first calculated for a fixed configuration of a background field, and then averaged with respect to a suitably defined effective action. We show that, after linearization of the energy dispersion at the Fermi surface, both methods yield for Fermi liquids exactly the same non-perturbative expression for the quasi-particle residue. However, in the case of non-Fermi liquid behavior the low-energy behavior of the Green's function predicted by the eikonal method can be erroneous. In particular, for the Tomonaga-Luttinger model the eikonal method neither reproduces the correct scaling behavior of the spectral function, nor predicts the correct location of its singularities.
\end{abstract}

PACS numbers: 72.10.Di, 05.30.Fk, 11.15-q,67.20+k 


\section{INTRODUCTION}

Recently a number of authors have developed generalizations of the bosonization method to arbitrary dimensions [1 10]. This approach is most suitable in interacting Fermi systems where the Fourier transform of the interaction is dominated by momentum transfers small compared with the Fermi momentum $k_{F}$. In this case the single-particle Green's function satisfies a non-trivial asymptotic Ward-identity [11, 12], which opens the way for a controlled summation of the entire perturbation series. Bosonization is one possible (and perhaps the most efficient) way to explicitly carry out this summation. Interactions that exhibit in Fourier space a strong maximum or even a singularity for small momentum transfers are long-range in real space. The experimentally perhaps most relevant interaction of this type is the effective current-current interaction due to the coupling between fermions and transverse gauge fields. Such a coupling appears in models for strongly correlated electron systems [13]. Moreover, effective current-current interactions are also generated by fluctuations of the statistical gauge field in the Chern-Simons theory for quantum Hall systems [14]. In Refs. [4.15] the gauge field problem has been studied via higher-dimensional bosonization. However, this approach has been criticized [16,12], because higher-dimensional bosonization relies (at least in its simplest form) on the linearization of the energy dispersion at the Fermi surface, and there exists evidence that in the gauge field problem the curvature of the Fermi surface cannot be neglected [16,12].

An alternative non-perturbative approach, which does not rely on the linearization of the energy dispersion, is the eikonal method. This approach has been developed in the 60 's to obtain non-perturbative results for the single-particle Green's function of field theories where the fermionic degrees of freedom are linearly coupled to another bosonic quantum field 17 20]. More recently, Khveshchenko and Stamp [21 have used this method to study the above mentioned gauge field problem in two dimensions. The strategy is to calculate first the Green's function $\mathcal{G}\left(\mathbf{r}, \mathbf{r}^{\prime}, t, t^{\prime} ;\{\mathbf{A}\}\right)$ of the fermions for fixed configuration of the gauge field $\mathbf{A}$, and then average the result with respect to the effective gauge

field action $\tilde{S}_{\text {eff }}\{\mathbf{A}\}$ to obtain the translationally invariant fermionic Green's function [22], $G\left(\mathbf{r}-\mathbf{r}^{\prime}, t-t^{\prime}\right)=\left\langle\mathcal{G}\left(\mathbf{r}, \mathbf{r}^{\prime}, t, t^{\prime} ;\{\mathbf{A}\}\right)\right\rangle_{\tilde{S}_{e f f}}$. To leading order in the eikonal expansion the averaging involves a trivial Gaussian integration, and gives rise to the usual Debye-Waller factor [21]. The functional integral approach to bosonization [23, 17, 10, 15] follows precisely the same strategy: The fermionic two-body interaction is first decoupled via a HubbardStratonovich transformation involving a space- and time-dependent auxiliary field; then the fermionic Green's function is calculated for a fixed configuration of the auxiliary field, and finally the result is averaged with respect to an effective action $S_{\text {eff }}$. Although the functional bosonization approach [23,7, 10,15] is formulated in Euclidean (i.e. imaginary) time while the eikonal expansion is by construction a Minkowski (i.e. real) time method, the similarity in both procedures suggests that, after proper analytic continuation, they should yield equivalent results for models with linearized energy dispersion. In the present paper we shall carefully examine this point, and show that in general this is not the case. 


\section{FUNCTIONAL BOSONIZATION}

In order to compare bosonization with the eikonal expansion, we shall in this section briefly describe our functional bosonization approach, focusing on the calculation of the single-particle Green's function. For a more detailed description, see Refs. [7 10]. To calculate Matsubara Green's function of the many-body system, we subdivide the degrees of freedom close to the Fermi surface into a finite number of boxes $K^{\alpha}$ labelled by an index $\alpha$, and decouple the interaction with the help of a Hubbard-Stratonovich transformation involving auxiliary fields associated with the $K^{\alpha}$. For our purpose it is sufficient to consider conventional density-density interactions, which can be decoupled via scalar fields $\phi^{\alpha}$. The generalization to gauge fields is straightforward and can be found in Refs. [10,15]. After the standard transformations [9,10] the Matsubara Green's function $G(k) \equiv G\left(\mathbf{k}, i \tilde{\omega}_{n}\right)$ can be exactly written as 24

$$
G(k)=\int \mathcal{D}\left\{\phi^{\alpha}\right\} \mathcal{P}\left\{\phi^{\alpha}\right\}[\hat{G}]_{k k} \equiv\left\langle[\hat{G}]_{k k}\right\rangle_{S_{e f f}}
$$

Here $\hat{G}^{-1}$ is an infinite matrix in momentum- and frequency space, with matrix elements given by the formal Dyson equation $\left[\hat{G}^{-1}\right]_{k k^{\prime}}=\left[\hat{G}_{0}^{-1}\right]_{k k^{\prime}}-[\hat{V}]_{k k^{\prime}}$, where $\hat{G}_{0}$ is the noninteracting Matsubara Green's function matrix, $\left[\hat{G}_{0}\right]_{k k^{\prime}}=\delta_{k k^{\prime}}\left[i \tilde{\omega}_{n}-\epsilon_{\mathbf{k}}+\mu\right]^{-1}$, and the generalized self-energy matrix $\hat{V}$ is $[\hat{V}]_{k k^{\prime}}=\sum_{\alpha} \Theta^{\alpha}(\mathbf{k}) V_{k-k^{\prime}}^{\alpha}$, with $V_{q}^{\alpha}=\frac{i}{\beta} \phi_{q}^{\alpha}$. Here $\beta$ is the inverse temperature, $\mu$ is the chemical potential, $\epsilon_{\mathbf{k}}$ is the non-interacting energy dispersion, and the cutoff function $\Theta^{\alpha}(\mathbf{k})$ is unity if $\mathbf{k}$ belongs to box $K^{\alpha}$, and vanishes otherwise. The normalized probability distribution $\mathcal{P}\left\{\phi^{\alpha}\right\}$ is

$$
\mathcal{P}\left\{\phi^{\alpha}\right\}=\frac{e^{-S_{e f f}\left\{\phi^{\alpha}\right\}}}{\int \mathcal{D}\left\{\phi^{\alpha}\right\} e^{-S_{e f f}\left\{\phi^{\alpha}\right\}}},
$$

where the effective action for the $\phi^{\alpha}$-field is of the form $S_{\text {eff }}\left\{\phi^{\alpha}\right\}=S_{2}\left\{\phi^{\alpha}\right\}+S_{k i n}\left\{\phi^{\alpha}\right\}$, with

$$
\begin{aligned}
S_{2}\left\{\phi^{\alpha}\right\} & =\frac{1}{2} \sum_{q} \sum_{\alpha \alpha^{\prime}}\left[\underline{\tilde{f}}_{q}^{-1}\right]^{\alpha \alpha^{\prime}} \phi_{-q}^{\alpha} \phi_{q}^{\alpha^{\prime}}, \\
S_{k i n}\left\{\phi^{\alpha}\right\} & =-\operatorname{Tr} \ln \left[1-\hat{G}_{0} \hat{V}\right] .
\end{aligned}
$$

Here $\underline{\tilde{f}}_{q}$ is a matrix in the patch indices, with matrix elements $\left[\underline{\tilde{f}}_{q}\right]^{\alpha \alpha^{\prime}}=\frac{\beta}{V} f_{q}^{\alpha \alpha^{\prime}}$, where $f_{q}^{\alpha \alpha^{\prime}}$ are the usual Landau interaction parameters, and $V$ is the volume of the system. The probability distribution $\mathcal{P}\left\{\phi^{\alpha}\right\}$ can be calculated perturbatively by expanding Eq. 1 in powers of the $\phi^{\alpha}$ field. The validity of this expansion is controlled by the generalized closed loop theorem, which is discussed in detail in Refs. [8,10]. At the level of the Gaussian approximation one obtains

$$
S_{k i n}\left\{\phi^{\alpha}\right\} \approx \frac{V}{2 \beta} \sum_{q} \sum_{\alpha \alpha^{\prime}} \Pi_{0}^{\alpha \alpha^{\prime}}(q) \phi_{-q}^{\alpha} \phi_{q}^{\alpha^{\prime}}
$$

where the "patch" polarization is given by 


$$
\begin{aligned}
\Pi_{0}^{\alpha \alpha^{\prime}}(q)= & -\frac{1}{2 \beta V} \sum_{k}\left[\Theta^{\alpha}(\mathbf{k}) \Theta^{\alpha^{\prime}}(\mathbf{k}+\mathbf{q}) G_{0}(k) G_{0}(k+q)\right. \\
& \left.+\Theta^{\alpha^{\prime}}(\mathbf{k}) \Theta^{\alpha}(\mathbf{k}-\mathbf{q}) G_{0}(k) G_{0}(k-q)\right]
\end{aligned}
$$

The leading correction to Eq.5 is given in Refs. 8, 10]. The calculation of the diagonal elements $[\hat{G}]_{k k}$ in Eq.1 is more difficult. Choosing the patches larger than the range of the interaction in momentum space, we may write [9,10] $[\hat{G}]_{k k}=\sum_{\alpha} \Theta^{\alpha}(\mathbf{k})\left[\hat{G}^{\alpha}\right]_{k k}$, where (after shifting $\mathbf{k}=\mathbf{k}^{\alpha}+\mathbf{q}, \mathbf{k}^{\prime}=\mathbf{k}^{\alpha}+\mathbf{q}^{\prime}$ ) the infinite matrix $\hat{G}^{\alpha}$ satisfies 24]

$$
\sum_{\tilde{q}^{\prime}}\left[\delta_{\tilde{q}, \tilde{q}^{\prime}}\left[G_{0}^{\alpha}(\tilde{q})\right]^{-1}-V_{\tilde{q}-\tilde{q}^{\prime}}^{\alpha}\right]\left[\hat{G}^{\alpha}\right]_{\tilde{q}^{\prime} \tilde{q}^{\prime \prime}}=\delta_{\tilde{q}, \tilde{q}^{\prime \prime}}
$$

Here $\left[G_{0}^{\alpha}(\tilde{q})\right]^{-1}=i \tilde{\omega}_{n}-\xi^{\alpha}(\mathbf{q})$, with $\xi^{\alpha}(\mathbf{q})=\epsilon_{\mathbf{k}^{\alpha}+\mathbf{q}}-\mu$. Defining $V^{\alpha}(\mathbf{r}, \tau)=\sum_{q} e^{i\left(\mathbf{q} \cdot \mathbf{r}-\omega_{m} \tau\right)} V_{q}^{\alpha}$ and

$$
\mathcal{G}^{\alpha}\left(\mathbf{r}, \mathbf{r}^{\prime}, \tau, \tau^{\prime}\right)=\frac{1}{\beta V} \sum_{\tilde{q} \tilde{q}^{\prime}} e^{i\left(\mathbf{q} \cdot \mathbf{r}-\tilde{\omega}_{n} \tau\right)} e^{-i\left(\mathbf{q}^{\prime} \cdot \mathbf{r}^{\prime}-\tilde{\omega}_{n^{\prime}} \tau^{\prime}\right)}\left[\hat{G}^{\alpha}\right]_{\tilde{q} \tilde{q}^{\prime}}
$$

it is easy to see that Eq.7 is equivalent with

$$
\left[-\partial_{\tau}-\xi^{\alpha}\left(\mathbf{P}_{\mathbf{r}}\right)-V^{\alpha}(\mathbf{r}, \tau)\right] \mathcal{G}^{\alpha}\left(\mathbf{r}, \mathbf{r}^{\prime}, \tau, \tau^{\prime}\right)=\delta\left(\mathbf{r}-\mathbf{r}^{\prime}\right) \delta^{*}\left(\tau-\tau^{\prime}\right)
$$

where $\mathbf{P}_{\mathbf{r}}=-i \nabla_{\mathbf{r}}$ is the momentum operator, and $\delta^{*}\left(\tau-\tau^{\prime}\right)=\frac{1}{\beta} \sum_{n} e^{-i \tilde{\omega}_{n}\left(\tau-\tau^{\prime}\right)}$. Eq.9 together with the Kubo-Martin-Schwinger (KMS) boundary conditions [25],

$$
\mathcal{G}^{\alpha}\left(\mathbf{r}, \mathbf{r}^{\prime}, \tau+\beta, \tau^{\prime}\right)=\mathcal{G}^{\alpha}\left(\mathbf{r}, \mathbf{r}^{\prime}, \tau, \tau^{\prime}+\beta\right)=-\mathcal{G}^{\alpha}\left(\mathbf{r}, \mathbf{r}^{\prime}, \tau, \tau^{\prime}\right)
$$

uniquely determine the Green's function. For linearized energy dispersion, $\xi^{\alpha}(\mathbf{q}) \approx \mathbf{v}^{\alpha} \cdot \mathbf{q}$, Eq.9 can be solved exactly by means of a trivial generalization of Schwinger's ansatz [26]. The result is $[7]$

$$
\begin{aligned}
\mathcal{G}^{\alpha}\left(\mathbf{r}, \mathbf{r}^{\prime}, \tau, \tau^{\prime}\right) & =G_{0}^{\alpha}\left(\mathbf{r}-\mathbf{r}^{\prime}, \tau-\tau^{\prime}\right) e^{\Phi^{\alpha}(\mathbf{r}, \tau)-\Phi^{\alpha}\left(\mathbf{r}^{\prime}, \tau^{\prime}\right)}, \\
G_{0}^{\alpha}(\mathbf{r}, \tau) & =\frac{1}{\beta V} \sum_{\tilde{q}} \frac{e^{i\left(\mathbf{q} \cdot \mathbf{r}-\tilde{\omega}_{n} \tau\right)}}{i \tilde{\omega}_{n}-\mathbf{v}^{\alpha} \cdot \mathbf{q}}, \\
\Phi^{\alpha}(\mathbf{r}, \tau) & =\sum_{q} \frac{e^{i\left(\mathbf{q} \cdot \mathbf{r}-\omega_{m} \tau\right)}}{i \omega_{m}-\mathbf{v}^{\alpha} \cdot \mathbf{q}} V_{q}^{\alpha} .
\end{aligned}
$$

Note that the bosonic Matsubara frequencies in Eq.13 insure that $\Phi^{\alpha}(\mathbf{r}, \tau+\beta)=\Phi^{\alpha}(\mathbf{r}, \tau)$, so that the ansatz 11 manifestly satisfies the KMS boundary condition 10. Gaussian averaging of Eq.11] with the effective action $S_{\text {eff }}\left\{\phi^{\alpha}\right\}$ given in Eqs. 3 and 5 yields

$$
\begin{gathered}
\left\langle\mathcal{G}^{\alpha}\left(\mathbf{r}, \mathbf{r}^{\prime}, \tau, \tau^{\prime}\right)\right\rangle_{S_{e f f}}=G_{0}^{\alpha}\left(\mathbf{r}-\mathbf{r}^{\prime}, \tau-\tau^{\prime}\right) e^{Q^{\alpha}\left(\mathbf{r}-\mathbf{r}^{\prime}, \tau-\tau^{\prime}\right)}, \\
Q^{\alpha}(\mathbf{r}, \tau)=\frac{1}{\beta V} \sum_{q} \frac{f_{q}^{R P A, \alpha}}{\left(i \omega_{m}-\mathbf{v}^{\alpha} \cdot \mathbf{q}\right)^{2}}\left[1-\cos \left(\mathbf{q} \cdot \mathbf{r}-\omega_{m} \tau\right)\right] .
\end{gathered}
$$


Here $f_{q}^{R P A, \alpha}$ is the screened interaction within random-phase approximation (RPA),

$$
f_{q}^{R P A, \alpha}=\left[\underline{f}_{q}\left[1+\underline{\Pi}_{0}(q) \underline{f}_{q}\right]^{-1}\right]^{\alpha \alpha}
$$

where underlined quantities are matrices in the patch indices. For the special case of the one-dimensional Tomonaga-Luttinger model [27] Eq.14 exactly reproduces the well-known bosonization result 23, 10.

\section{THE EIKONAL EXPANSION}

An obvious disadvantage of the procedure outlined in the previous section is that the Schwinger solution in Eqs.11-13 is only valid for linearized energy dispersion. In general, the effective energy dispersion in box $K^{\alpha}$ is of the form $\epsilon_{\mathbf{k}^{\alpha}+\mathbf{q}}=\epsilon_{\mathbf{k}^{\alpha}}+\mathbf{v}^{\alpha} \cdot \mathbf{q}+\frac{\mathbf{q}^{2}}{2 m^{\alpha}}$. The eikonal method can in principle handle the quadratic (curvature) term in a systematic way. However, this method cannot be directly compared with our functional bosonization approach, because it is by construction a real time method. Let us briefly summarize the main steps. For simplicity we focus here on the retarded Green's function for fixed real time background field $\tilde{V}^{\alpha}(\mathbf{r}, t)$, which is defined via the differential equation

$$
\left[i \partial_{t}-\xi^{\alpha}\left(\mathbf{P}_{\mathbf{r}}\right)-\tilde{V}^{\alpha}(\mathbf{r}, t)\right] \mathcal{G}_{r e t}^{\alpha}\left(\mathbf{r}, \mathbf{r}^{\prime}, t, t^{\prime}\right)=\delta\left(\mathbf{r}-\mathbf{r}^{\prime}\right) \delta\left(t-t^{\prime}\right)
$$

with the boundary condition that $\mathcal{G}_{\text {ret }}^{\alpha}\left(\mathbf{r}, \mathbf{r}^{\prime}, t, t^{\prime}\right)=0$ for $t-t^{\prime}<0$. In Sec. $\mathbb{\square}$ we shall discuss how to obtain for the leading term in the eikonal expansion the corresponding time-ordered Green's function, which is usually calculated in diagrammatic perturbation theory. The eikonal expansion is a systematic method for solving partial differential equations of the type 17. This approach has been developed many years ago [17 20]. In this section we shall briefly outline how Eq.17 can be solved with this method, following mainly Ref. 19.

It is instructive to study first the solution of Eq.17 without external potential, i.e. for $\tilde{V}^{\alpha}(\mathbf{r}, t)=0$. Then the retarded propagator is given by [22]

$$
G_{r e t, 0}^{\alpha}\left(\mathbf{r}-\mathbf{r}^{\prime}, t-t^{\prime}\right)=\int \frac{d \mathbf{q}}{(2 \pi)^{d}} \int \frac{d \omega}{2 \pi} e^{i\left[\mathbf{q} \cdot\left(\mathbf{r}-\mathbf{r}^{\prime}\right)-\omega\left(t-t^{\prime}\right)\right]} G_{r e t, 0}^{\alpha}(\mathbf{q}, \omega)
$$

with

$$
G_{r e t, 0}^{\alpha}(\mathbf{q}, \omega)=\frac{1}{\omega-\xi^{\alpha}(\mathbf{q})+i 0^{+}}=-i \int_{0}^{\infty} d t^{\prime \prime} e^{i t^{\prime \prime}\left[\omega-\xi^{\alpha}(\mathbf{q})+i 0^{+}\right]}
$$

As will be evident shortly, the representation of the propagator in terms of an integral over an auxiliary time $t^{\prime \prime}$ plays an important role in the eikonal approach. Note, however, that we can exactly eliminate the auxiliary integral by substituting Eq.19 into Eq.18 and doing first the frequency integration, using $\int \frac{d \omega}{2 \pi} e^{-i \omega\left(t-t^{\prime}-t^{\prime \prime}\right)}=\delta\left(t-t^{\prime}-t^{\prime \prime}\right)$. Then the $t^{\prime \prime}$-integral is trivial, and we obtain

$$
G_{r e t, 0}^{\alpha}\left(\mathbf{r}-\mathbf{r}^{\prime}, t-t^{\prime}\right)=-i \Theta\left(t-t^{\prime}\right) \int \frac{d \mathbf{q}}{(2 \pi)^{d}} e^{i\left[\mathbf{q} \cdot\left(\mathbf{r}-\mathbf{r}^{\prime}\right)-\xi^{\alpha}(\mathbf{q})\left(t-t^{\prime}\right)\right]}
$$


If we linearize the energy dispersion (i.e. set $m^{\alpha}=\infty$, as in conventional bosonization), then the q-integration can be performed exactly, with the result

$$
G_{r e t, 0}^{\alpha}\left(\mathbf{r}-\mathbf{r}^{\prime}, t-t^{\prime}\right)=-i \Theta\left(t-t^{\prime}\right) \delta^{(d)}\left(\mathbf{r}-\mathbf{r}^{\prime}-\mathbf{v}^{\alpha}\left(t-t^{\prime}\right)\right) \quad, \quad m^{\alpha}=\infty .
$$

Let us now consider the case $\tilde{V}^{\alpha}(\mathbf{r}, t) \neq 0$. The starting point is the transformation of Eq.17 to the mixed representation by Fourier transforming it with respect to the difference variables $\mathbf{r}-\mathbf{r}^{\prime}$ and $t-t^{\prime}[19]$. Defining

$$
\mathcal{G}_{\text {ret }}^{\alpha}\left(\mathbf{r}, \mathbf{r}^{\prime}, t, t^{\prime}\right)=\int \frac{d \mathbf{q}}{(2 \pi)^{d}} \int \frac{d \omega}{2 \pi} e^{i\left[\mathbf{q} \cdot\left(\mathbf{r}-\mathbf{r}^{\prime}\right)-\omega\left(t-t^{\prime}\right)\right]} \mathcal{G}_{r e t}^{\alpha}(\mathbf{r}, t ; \mathbf{q}, \omega)
$$

it is easy to see that Eq.17 reduces to

$$
\left[i \partial_{t}-\xi^{\alpha}\left(\mathbf{P}_{\mathbf{r}}\right)-\tilde{V}^{\alpha}(\mathbf{r}, t)+\omega-\xi^{\alpha}(\mathbf{q})\right] \mathcal{G}_{r e t}^{\alpha}(\mathbf{r}, t ; \mathbf{q}, \omega)=1
$$

The formal solution of this equation can be written as 19

$$
\mathcal{G}_{\text {ret }}^{\alpha}(\mathbf{r}, t ; \mathbf{q}, \omega)=-i \int_{0}^{\infty} d t^{\prime \prime} e^{i t^{\prime \prime}\left[\omega-\xi^{\alpha}(\mathbf{q})+i 0^{+}\right]} Y^{\alpha}\left(t^{\prime \prime} ; \mathbf{r}, t\right)
$$

where the auxiliary function $Y^{\alpha}\left(t^{\prime \prime} ; \mathbf{r}, t\right)$ satisfies the partial differential equation

$$
\left[i \partial_{t^{\prime \prime}}+i \partial_{t}-\xi^{\alpha}\left(\mathbf{P}_{\mathbf{r}}\right)-\tilde{V}^{\alpha}(\mathbf{r}, t)\right] Y^{\alpha}\left(t^{\prime \prime} ; \mathbf{r}, t\right)=0
$$

with boundary condition $Y^{\alpha}(0 ; \mathbf{r}, t)=1$. The correctness of Eq.24 is easily verified by applying the differential operator in Eq.23 to the right-hand side of Eq.24 and integrating by parts. Note also that without external potential the unique solution of Eq.25 with the correct boundary condition is $Y^{\alpha}\left(t^{\prime \prime} ; \mathbf{r}, t\right)=1$, so that Eq.24 reduces to the right-hand side of Eq.19. It is convenient to parametrize the solution of Eq.25 in the form $Y^{\alpha}\left(t^{\prime \prime} ; \mathbf{r}, t\right)=e^{F^{\alpha}\left(t^{\prime \prime} ; \mathbf{r}, t\right)}$. The equivalent differential equation for $F^{\alpha}$ is then

$$
\left[i \partial_{t^{\prime \prime}}+i \partial_{t}-\xi^{\alpha}\left(\mathbf{P}_{\mathbf{r}}\right)\right] F^{\alpha}\left(t^{\prime \prime} ; \mathbf{r}, t\right)=\tilde{V}^{\alpha}(\mathbf{r}, t)+\frac{\left[\mathbf{P}_{\mathbf{r}} F^{\alpha}\left(t^{\prime \prime} ; \mathbf{r}, t\right)\right]^{2}}{2 m^{\alpha}},
$$

with boundary condition $F^{\alpha}(0 ; \mathbf{r}, t)=0$. This non-linear partial differential equation cannot be solved exactly. However, we can obtain the solution as expansion in powers of the external potential $\tilde{V}^{\alpha}$ [19. Substituting the ansatz $F^{\alpha}\left(t^{\prime \prime} ; \mathbf{r}, t\right)=\sum_{n=1}^{\infty} F_{n}^{\alpha}\left(t^{\prime \prime} ; \mathbf{r}, t\right)$ into Eq.26 (where $F_{n}^{\alpha}$ is by assumption of order $\left.\left(\tilde{V}^{\alpha}\right)^{n}\right)$, we have

$$
\left[i \partial_{t^{\prime \prime}}+i \partial_{t}-\xi^{\alpha}\left(\mathbf{P}_{\mathbf{r}}\right)\right] F_{n}^{\alpha}\left(t^{\prime \prime} ; \mathbf{r}, t\right)=\tilde{V}_{n}^{\alpha}\left(t^{\prime \prime} ; \mathbf{r}, t\right) \quad, \quad n=1,2, \ldots
$$

with $\tilde{V}_{1}^{\alpha}\left(t^{\prime \prime} ; \mathbf{r}, t\right)=\tilde{V}^{\alpha}(\mathbf{r}, t)$ (independent of $\left.t^{\prime \prime}\right)$, and

$$
\tilde{V}_{n}^{\alpha}\left(t^{\prime \prime} ; \mathbf{r}, t\right)=\frac{1}{2 m^{\alpha}} \sum_{n^{\prime}=1}^{n-1}\left[\mathbf{P}_{\mathbf{r}} F_{n^{\prime}}^{\alpha}\left(t^{\prime \prime} ; \mathbf{r}, t\right)\right] \cdot\left[\mathbf{P}_{\mathbf{r}} F_{n-n^{\prime}}^{\alpha}\left(t^{\prime \prime} ; \mathbf{r}, t\right)\right] \quad, \quad n=2,3, \ldots .
$$

Eq.27 is easily solved by means of the Green's function of the differential operator on the left-hand side. The solution with the correct boundary condition can be written as

$$
F_{n}^{\alpha}\left(t^{\prime \prime} ; \mathbf{r}, t\right)=-i \int_{0}^{t^{\prime \prime}} d s e^{i\left[i \partial_{t}-\xi^{\alpha}\left(\mathbf{P}_{\mathbf{r}}\right)\right]\left(t^{\prime \prime}-s\right)} \tilde{V}_{n}^{\alpha}(s ; \mathbf{r}, t)
$$

This completes the formal solution of the problem. 


\section{FORMAL SIMILARITIES BETWEEN BOSONIZATION AND THE EIKONAL EXPANSION FOR LINEARIZED ENERGY DISPERSION}

Suppose we truncate the eikonal expansion at the first order, $F^{\alpha}\left(t^{\prime \prime} ; \mathbf{r}, t\right) \approx F_{1}^{\alpha}\left(t^{\prime \prime} ; \mathbf{r}, t\right)$. This approximation becomes exact in the limit $m^{\alpha}=\infty$ (i.e. for linearized energy dispersion), because then all higher order potentials $\tilde{V}_{n}^{\alpha}$ with $n \geq 2$ in Eq.27 vanish identically. For finite $m^{\alpha}$, we expect that the relevant small parameter which controls this approximation is proportional to the product of $1 / m^{\alpha}$ times the typical strength of the effective screened interaction [8, 10]. The function $F_{1}^{\alpha}\left(t^{\prime \prime} ; \mathbf{r}, t\right)$ is easily calculated by decomposing $\tilde{V}^{\alpha}(\mathbf{r}, t)$ into its Fourier components,

$$
\tilde{V}^{\alpha}(\mathbf{r}, t)=\int \frac{d \mathbf{q}}{(2 \pi)^{d}} \int \frac{d \omega}{2 \pi} e^{i[\mathbf{q} \cdot \mathbf{r}-\omega t]} \tilde{V}^{\alpha}(\mathbf{q}, \omega)
$$

and using

$$
e^{i\left[i \partial_{t}-\xi^{\alpha}\left(\mathbf{P}_{\mathbf{r}}\right)\right]\left(t^{\prime \prime}-s\right)} e^{i[\mathbf{q} \cdot \mathbf{r}-\omega t]}=e^{i\left[\omega-\xi^{\alpha}(\mathbf{q})\right]\left(t^{\prime \prime}-s\right)} e^{i[\mathbf{q} \cdot \mathbf{r}-\omega t]}
$$

After performing the $s$-integration in Eq.29 we obtain

$$
F_{1}^{\alpha}\left(t^{\prime \prime} ; \mathbf{r}, t\right)=\int \frac{d \mathbf{q}}{(2 \pi)^{d}} \int \frac{d \omega}{2 \pi} e^{i[\mathbf{q} \cdot \mathbf{r}-\omega t]} \frac{\tilde{V}^{\alpha}(\mathbf{q}, \omega)}{\omega-\xi^{\alpha}(\mathbf{q})}\left[1-e^{i\left[\omega-\xi^{\alpha}(\mathbf{q}) t^{\prime \prime}\right.}\right]
$$

The leading eikonal result for the retarded Green's function in a given external potential is then

$$
\begin{aligned}
& \mathcal{G}_{r e t, 1}^{\alpha}\left(\mathbf{r}, \mathbf{r}^{\prime}, t, t^{\prime}\right)=\int \frac{d \mathbf{q}}{(2 \pi)^{d}} \int \frac{d \omega}{2 \pi} e^{i\left[\mathbf{q} \cdot\left(\mathbf{r}-\mathbf{r}^{\prime}\right)-\omega\left(t-t^{\prime}\right)\right]} \mathcal{G}_{r e t, 1}^{\alpha}(\mathbf{r}, t ; \mathbf{q}, \omega) \\
& \mathcal{G}_{r e t, 1}^{\alpha}(\mathbf{r}, t ; \mathbf{q}, \omega)=-i \int_{0}^{\infty} d t^{\prime \prime} e^{i t^{\prime \prime}\left[\omega-\xi^{\alpha}(\mathbf{q})+i 0^{+}\right]} e^{F_{1}^{\alpha}\left(t^{\prime \prime} ; \mathbf{r}, t\right)}
\end{aligned}
$$

We now show that for linearized energy dispersion (i.e. for $m^{\alpha}=\infty$ ) Eqs.33 and 34 have exactly the same structure as the Schwinger solution given in Eqs.11.13. At the first sight this is not at all clear, because the eikonal result in Eqs.33 and 34 involves an additional integration over the auxiliary time $t^{\prime \prime}$. In the work of Khveshchenko and Stamp [21] the $t^{\prime \prime}$ integration has been performed by means of the saddle-point method. However, to see the connection with bosonization, we eliminate the auxiliary variable $t^{\prime \prime}$ from Eq. 34. Therefore we simply repeat the manipulations leading to Eq.20 and first perform the $\omega$-integration in Eq.33. As before, this gives rise to a factor of $\delta\left(t-t^{\prime}-t^{\prime \prime}\right)$. The $t^{\prime \prime}$-integration is then trivial, and we obtain

$$
\mathcal{G}_{r e t, 1}^{\alpha}\left(\mathbf{r}, \mathbf{r}^{\prime}, t, t^{\prime}\right)=G_{r e t, 0}^{\alpha}\left(\mathbf{r}-\mathbf{r}^{\prime}, t-t^{\prime}\right) e^{F_{1}^{\alpha}\left(t-t^{\prime} ; \mathbf{r}, t\right)}
$$

with $G_{r e t, 0}^{\alpha}\left(\mathbf{r}-\mathbf{r}^{\prime}, t-t^{\prime}\right)$ given in Eq.20. Still, the exponential factor in Eq.35 does not have the same structure as the corresponding factor in Eq.11. However, for $m^{\alpha}=\infty$ the function $G_{r e t, 0}^{\alpha}\left(\mathbf{r}-\mathbf{r}^{\prime}, t-t^{\prime}\right)$ is proportional to $\delta^{(d)}\left(\mathbf{r}-\mathbf{r}^{\prime}-\mathbf{v}^{\alpha}\left(t-t^{\prime}\right)\right)$ (see Eq.21), so that we may replace $\mathbf{v}^{\alpha}\left(t-t^{\prime}\right) \rightarrow \mathbf{r}-\mathbf{r}^{\prime}$ in the expression for $F_{1}^{\alpha}\left(t-t^{\prime} ; \mathbf{r}, t\right)$ in Eq.35, 


$$
\begin{aligned}
e^{i[\mathbf{q} \cdot \mathbf{r}-\omega t]}\left[1-e^{i\left[\omega-\xi^{\alpha}(\mathbf{q})\right]\left(t-t^{\prime}\right)}\right] & =e^{i[\mathbf{q} \cdot \mathbf{r}-\omega t]}\left[1-e^{i \omega\left(t-t^{\prime}\right)-i \mathbf{q} \cdot \mathbf{v}^{\alpha}\left(t-t^{\prime}\right)}\right] \\
\rightarrow e^{i[\mathbf{q} \cdot \mathbf{r}-\omega t]}\left[1-e^{i\left[\omega\left(t-t^{\prime}\right)-\mathbf{q} \cdot\left(\mathbf{r}-\mathbf{r}^{\prime}\right)\right]}\right] & =e^{i[\mathbf{q} \cdot \mathbf{r}-\omega t]}-e^{i\left[\mathbf{q} \cdot \mathbf{r}^{\prime}-\omega t^{\prime}\right]} .
\end{aligned}
$$

Then it is easy to see that Eq. 35 can also be written as

$$
\mathcal{G}_{r e t, 1}^{\alpha}\left(\mathbf{r}, \mathbf{r}^{\prime}, t, t^{\prime}\right)=G_{r e t, 0}^{\alpha}\left(\mathbf{r}-\mathbf{r}^{\prime}, t-t^{\prime}\right) e^{\Phi_{1}^{\alpha}(\mathbf{r}, t)-\Phi_{1}^{\alpha}\left(\mathbf{r}^{\prime}, t^{\prime}\right)}
$$

with

$$
\Phi_{1}^{\alpha}(\mathbf{r}, t)=\int \frac{d \mathbf{q}}{(2 \pi)^{d}} \int \frac{d \omega}{2 \pi} e^{i[\mathbf{q} \cdot \mathbf{r}-\omega t]} \frac{\tilde{V}^{\alpha}(\mathbf{q}, \omega)}{\omega-\mathbf{v}^{\alpha} \cdot \mathbf{q}}
$$

The formal similarity to Eqs.11.13 is now evident. Note, however, that the imaginary time finite-temperature Green's function discussed in Sec.II and the real time retarded Green's function discussed here are not related in a trivial way: only after Fourier transformation into the frequency domain there exists a simple relation via the spectral function.

\section{AVERAGING IN MINKOWSKI TIME: HOW GOOD IS THE EIKONAL EXPANSION FOR LINEARIZED ENERGY DISPERSION?}

In spite of the formal similarity between the real time eikonal result in Eq.37 and the imaginary time bosonization solution in Eq.11, after the averaging procedure the final expressions for the Green's function of the many-body system are not equivalent. To average Eq.34, one should keep in mind that by construction functional averaging always generates time-ordered correlation functions [28]. Hence, in order to properly define the averaging procedure, we should replace the retarded Green's function in Eq.34 by the corresponding time-ordered Green's function before averaging with respect to the effective action $\tilde{S}_{\text {eff }}$ of the background field. This is easily done by going back to Eq.19 and choosing the limit for the $t^{\prime \prime}$-integration such that the integral for the time-ordered Green's function is convergent. With the notation $s_{\omega}=\operatorname{sign} \omega$ we have

$$
G_{0}^{\alpha}(\mathbf{q}, \omega)=\frac{1}{\omega-\xi^{\alpha}(\mathbf{q})+i 0^{+} s_{\omega}}=-i \int_{0}^{\infty s_{\omega}} d t^{\prime \prime} e^{i t^{\prime \prime}\left[\omega-\xi^{\alpha}(\mathbf{q})+i 0^{+} s_{\omega}\right]} .
$$

Using the fact that functional averaging restores translational invariance in space and time, it is easy to see that after averaging the leading eikonal result for the time-ordered Green's function reads

$$
G_{1}^{\alpha}(\mathbf{q}, \omega)=-i \int_{0}^{\infty s_{\omega}} d t^{\prime \prime} e^{i t^{\prime \prime}\left[\omega-\xi^{\alpha}(\mathbf{q})+i 0^{+} s_{\omega}\right]}\left\langle e^{F_{1}^{\alpha}\left(t^{\prime \prime} ; 0,0\right)}\right\rangle_{\tilde{S}_{e f f}} .
$$

At the level of the Gaussian approximation the functional average in Eq.40 generates the usual Debye-Waller factor, with propagator given by the RPA-interaction [21]. Thus,

$$
G_{1}^{\alpha}(\mathbf{q}, \omega)=-i \int_{0}^{\infty s_{\omega}} d t^{\prime \prime} e^{i t^{\prime \prime}\left[\omega-\xi^{\alpha}(\mathbf{q})+i 0^{+} s_{\omega}\right]} e^{Q_{1}^{\alpha}\left(t^{\prime \prime}\right)}
$$

with 


$$
Q_{1}^{\alpha}\left(t^{\prime \prime}\right)=\int \frac{d \mathbf{q}^{\prime}}{(2 \pi)^{d}} \int \frac{d \omega^{\prime}}{2 \pi i} f^{R P A}\left(\mathbf{q}^{\prime}, \omega^{\prime}\right) \frac{1-\cos \left[\left(\omega^{\prime}-\xi^{\alpha}\left(\mathbf{q}^{\prime}\right)\right) t^{\prime \prime}\right]}{\left[\omega^{\prime}-\xi^{\alpha}\left(\mathbf{q}^{\prime}\right)\right]^{2}} .
$$

For simplicity we have assumed a patch- and frequency-independent bare interaction $f_{\mathbf{q}}$, so that the screened interaction can be identified with the usual RPA interaction, $f^{R P A}(\mathbf{q}, \omega)=$ $f_{\mathbf{q}}\left[1+\Pi_{0}(\mathbf{q}, \omega) f_{\mathbf{q}}\right]^{-1}$, where $\Pi_{0}(\mathbf{q}, \omega)$ is the Lindhard function [29]. In Eq.42 we have called the integration variables $\mathbf{q}^{\prime}$ and $\omega^{\prime}$ to avoid confusion with the external labels $\mathbf{q}$ and $\omega$ in Eq.41.

According to Eq.41 the spectral function $-\frac{1}{\pi} \operatorname{Im} G_{1}^{\alpha}\left(\mathbf{q}, \omega+i 0^{+}\right)$of our interacting manybody system can be obtained via a one-dimensional Fourier transformation. Furthermore, except for the trivial factor of $s_{\omega}$, the spectral function depends exclusively on the combination $\omega-\xi^{\alpha}(\mathbf{q})$. In particular, for linearized energy dispersion $\xi^{\alpha}(\mathbf{q})=\mathbf{v}^{\alpha} \cdot \mathbf{q}$ (where the eikonal expansion truncates at the first order, so that Eq.37 is the exact solution of Eq.17) the spectral function is according to Eq.41 a combination of the variable $\omega-\mathbf{v}^{\alpha} \cdot \mathbf{q}$ only. The well-known bosonization result for the Green's function of the Tomonaga-Luttinger model [27] (i.e. one-dimensional electrons with long-range interactions and exactly linear energy dispersion) shows that in one dimension this result cannot be correct [30]! The spectral function of the Tomonaga-Luttinger model exhibits a more complicated dependence on $\mathbf{q}$ and $\omega$ [31]. One possibility to cure this problem might be to choose some effective $\omega-$ or q-dependent cutoffs for the integration limits in Eq.42 [32]. However, such a procedure seems to require a certain amount of intuition and knowledge about the final result, and is certainly not satisfactory from the formal point of view.

The obvious question is now whether the spectral function calculated from the real time eikonal expansion given in Eqs.41 and 42 agrees at least approximately with the spectral function calculated from the imaginary time bosonization result given in Eqs.14 and 15. To clarify this point, it is useful to rewrite Eq.42 with the help of the dynamic structure factor [33], which is related to the RPA interaction via [10]

$$
f^{R P A}\left(\mathbf{q}^{\prime}, \omega^{\prime}\right)=f_{\mathbf{q}^{\prime}}-f_{\mathbf{q}^{\prime}}^{2} \int_{0}^{\infty} d \omega S^{R P A}\left(\mathbf{q}^{\prime}, \omega\right)\left[\frac{1}{\omega-\omega^{\prime}}+\frac{1}{\omega+\omega^{\prime}}\right] .
$$

Substituting this expression into Eq.42, it is obvious that we encounter poles on the real $\omega^{\prime}$-axis, which must be regularized by an appropriate deformation of the integration contour in the complex $\omega^{\prime}$-plane. The correct contour is easily determined from the requirement that the expansion of the factor $e^{Q_{1}^{\alpha}\left(t^{\prime \prime}\right)}$ in Eq.41 should reproduce the lowest order perturbation theory for the time-ordered Green's function [34]. It is easy to see that this implies that we use the dashed contour shown in Fig.1, which describes time-ordering and corresponds to the regularization $\omega^{\prime} \rightarrow \omega^{\prime}+i 0^{+} \operatorname{sign} \omega^{\prime}$ in Eq.43. The $\omega^{\prime}$-integral can then be carried out via contour integration. We obtain $Q_{1}^{\alpha}\left(t^{\prime \prime}\right)=R_{1}^{\alpha}-S_{1}^{\alpha}\left(t^{\prime \prime}\right)$, with

$$
\begin{gathered}
R_{1}^{\alpha}=-\int \frac{d \mathbf{q}}{(2 \pi)^{d}} f_{\mathbf{q}}^{2} \int_{0}^{\infty} d \omega \frac{S^{R P A}(\mathbf{q}, \omega)}{\left(\omega+\left|\mathbf{v}^{\alpha} \cdot \mathbf{q}\right|\right)^{2}} \\
S_{1}^{\alpha}\left(t^{\prime \prime}\right)=\frac{i t^{\prime \prime}}{2} \int \frac{d \mathbf{q}}{(2 \pi)^{d}} f^{R P A}\left(\mathbf{q}, \mathbf{v}^{\alpha} \cdot \mathbf{q}\right)-\int \frac{d \mathbf{q}}{(2 \pi)^{d}} f_{\mathbf{q}}^{2} \int_{0}^{\infty} d \omega \frac{S^{R P A}(\mathbf{q}, \omega)}{\left(\omega+\left|\mathbf{v}^{\alpha} \cdot \mathbf{q}\right|\right)^{2}} \\
\times\left\{\frac{e^{-i \omega t^{\prime \prime}}\left[\left(\omega^{2}+\left(\mathbf{v}^{\alpha} \cdot \mathbf{q}\right)^{2}\right) \cos \left(\mathbf{v}^{\alpha} \cdot \mathbf{q} t^{\prime \prime}\right)+2 i \omega \mathbf{v}^{\alpha} \cdot \mathbf{q} \sin \left(\mathbf{v}^{\alpha} \cdot \mathbf{q} t^{\prime \prime}\right)\right]-2 \omega\left|\mathbf{v}^{\alpha} \cdot \mathbf{q}\right|}{\left(\omega-\left|\mathbf{v}^{\alpha} \cdot \mathbf{q}\right|\right)^{2}}\right\}
\end{gathered}
$$


It is easy to show that the term $R_{1}^{\alpha}$ agrees exactly with the zero-temperature limit of the space- and time-independent contribution to $Q^{\alpha}(\mathbf{r}, \tau)$ in Eq.15,

$$
R_{1}^{\alpha}=\lim _{\beta \rightarrow \infty} \lim _{V \rightarrow \infty} \frac{1}{\beta V} \sum_{q} \frac{f_{q}^{R P A, \alpha}}{\left(i \omega_{m}-\mathbf{v}^{\alpha} \cdot \mathbf{q}\right)^{2}} .
$$

Because in a Fermi liquid the quantity $e^{R_{1}^{\alpha}}$ can be identified with the quasi-particle residue [7.10], it is clear that for Fermi liquids the real time eikonal approach and functional bosonization yield exactly the same non-perturbative result for the quasi-particle residue. The proper deformation of the contour into the complex $\omega^{\prime}$-plane was crucial to obtain this result. If we had directly averaged the eikonal result for the retarded Green's function in Eq.34 (choosing the retarded contour shown in Fig.(1), we would have obtained incorrectly $R_{1}^{\alpha}=0$.

The time-dependent contribution $S_{1}^{\alpha}\left(t^{\prime \prime}\right)$ cannot be directly compared with a corresponding term in Eq.15. To examine possible discrepancies with bosonization, let us explicitly calculate $Q_{1}^{\alpha}\left(t^{\prime \prime}\right)$ for the spinless Tomonaga-Luttinger model [27] with interaction parameters $g_{4}(\mathbf{q})=g_{2}(\mathbf{q})=f_{\mathbf{q}}$. In this case the patch index $\alpha= \pm$ labels the two Fermi points, and the dynamic structure factor is simply $S^{R P A}(\mathbf{q}, \omega)=Z_{\mathbf{q}} \delta\left(\omega-\omega_{\mathbf{q}}\right)$, with residue $Z_{\mathbf{q}}=\frac{|\mathbf{q}|}{2 \pi \sqrt{1+F_{\mathbf{q}}}}$ and collective mode $\omega_{\mathbf{q}}=\sqrt{1+F_{\mathbf{q}}} v_{F}|\mathbf{q}|$. Here $F_{\mathbf{q}}=f_{\mathbf{q}} /\left(\pi v_{F}\right)$ is the relevant dimensionless coupling. (It is understood that in $d=1$ the vector $\mathbf{q}$ has only one component.) It is also easy to show [10] that in this case $f^{R P A}\left(\mathbf{q}, \mathbf{v}^{\alpha} \cdot \mathbf{q}\right)=0$, so that the first term in Eq.45 vanishes. Because of the $\delta$-function dynamic structure factor, the $\omega$-integration in Eq.45 is trivial. To perform the remaining (one-dimensional) q-integral, we adopt the usual procedure [30] of replacing $f_{\mathbf{q}} \rightarrow f_{0}$ in the integrand and multiplying the integrand by an ultraviolet cutoff $e^{-|\mathbf{q}| / q_{c}}$. The $\mathbf{q}$-integration is then elementary and we obtain after straightforward algebra

$$
Q_{1}^{\alpha}\left(t^{\prime \prime}\right)=-\left(1+\frac{\gamma}{2}\right) \ln \left[1+i\left(\tilde{v}_{F}-v_{F}\right) q_{c} t^{\prime \prime}\right]-\frac{\gamma}{2} \ln \left[1+i\left(\tilde{v}_{F}+v_{F}\right) q_{c} t^{\prime \prime}\right],
$$

where

$$
\gamma=\frac{F_{0}^{2}}{2 \sqrt{1+F_{0}}\left(\sqrt{1+F_{0}}+1\right)^{2}}
$$

is the well-known anomalous dimension of the Tomonaga-Luttinger model, and $\tilde{v}_{F}=$ $\sqrt{1+F_{0}} v_{F}$ is the renormalized Fermi velocity. For $\omega>0$ the eikonal result for the spectral function of the spinless Tomonaga-Luttinger model can then be written as

$$
A^{\alpha}(\mathbf{q}, \omega)=\frac{1}{\pi} \operatorname{Re} \int_{0}^{\infty} d t^{\prime \prime} \frac{e^{i t^{\prime \prime}\left[\omega-\mathbf{v}^{\alpha} \cdot \mathbf{q}+i 0^{+}\right]}}{\left[1+i\left(\tilde{v}_{F}-v_{F}\right) q_{c} t^{\prime \prime}\right]^{1+\gamma / 2}\left[1+i\left(\tilde{v}_{F}+v_{F}\right) q_{c} t^{\prime \prime}\right]^{\gamma / 2}} .
$$

Note that the integrand vanishes as $\left(t^{\prime \prime}\right)^{1+\gamma}$ for large $t^{\prime \prime}$. Hence, for any finite interaction the integral in Eq.49 is convergent, and we may omit the convergence factor $i 0^{+}$in the exponent. Following Dzyaloshinksii and Larkin [30], we find that for small $\left|\omega-\mathbf{v}^{\alpha} \cdot \mathbf{q}\right|$ and $0<\gamma<1$ to leading order $A^{\alpha}(\mathbf{q}, \omega) \sim A_{0}^{\alpha}+A_{1}^{\alpha}\left|\omega-\mathbf{v}^{\alpha} \cdot \mathbf{q}\right|^{\gamma}$, where $A_{0}^{\alpha}$ and $A_{1}^{\alpha}$ are finite complex numbers. This is in striking disagreement with the well-known bosonization result [30], which predicts for $0<\gamma<1$ a singularity of the type $A^{\alpha}(\mathbf{q}, \omega) \propto\left|\omega-\sqrt{1+F_{0}} \mathbf{v}^{\alpha} \cdot \mathbf{q}\right|^{\gamma-1}$. Thus, the real time eikonal method neither reproduces the correct scaling behavior of the spectral function, nor does it predict the correct location of its singularities. 


\section{CONCLUSION}

In this work we have compared the real time eikonal method with functional bosonization. In both methods the translationally invariant Green's function $G$ of the many-body system is obtained by calculating first the Green's function $\mathcal{G}$ in a given background field, and then averaging the result with respect to a suitably defined Gaussian effective action, $G=\langle\mathcal{G}\rangle$. Although both methods produce very similar non-perturbative expressions for $\mathcal{G}$, the final results for the functinal average $\langle\mathcal{G}\rangle$ are certainly not equivalent. In particular, in the case of the one-dimensional Tomonaga-Luttinger model the real time eikonal method does not even reproduce the correct scaling behavior of the Green's function. We suspect that this shortcoming of the eikonal approach is related to the averaging in Minkowski time, which cannot be interpreted as averaging over an ordinary probability distribution. On the other hand, the imaginary time averaging performed in functional bosonization is mathematically well defined, and leads to an expression for the Green's function which is exactly equivalent with the result obtained via operator bosonization [3,5].

Because even at the level of the linearized theory the real time eikonal expansion can lead to incorrect results, we conclude that this method is not a good starting point for studying the effects associated with the curvature of the Fermi surface on the low-energy behavior of the Green's function of non-Fermi liquids. Inclusion of curvature effects is of particular importance in connection with the gauge field problem. It is therefore highly desirable to study the effect of the non-linear terms in the energy dispersion on the leading (linearized) bosonization expression for the Green's function entirely within the framework of the imaginary time approach described in Sec.II. Work along these lines is in progress.

\section{ACKNOWLEDGEMENTS}

I would like to thank K. Schönhammer for discussions and comments on the manuscript. I have also profited from discussions with G. E. Castilla, D. V. Khveshchenko, and V. Meden. 


\section{REFERENCES}

[1] A. Luther, Phys. Rev. B 19, 320 (1979).

[2] F. D. M. Haldane, Helv. Phys. Acta. 65, 152 (1992); in Perspectives in Many-Particle Physics, Proceedings of the International School of Physics "Enrico Fermi", Course 121, (North Holland, Amsterdam, 1994).

[3] A. Houghton and J. B. Marston, Phys. Rev. B 48, 7790 (1993); A. Houghton, H. J. Kwon, and J. B. Marston, Phys. Rev. B 50, 1351 (1994); A. Houghton, H. J. Kwon, J. B. Marston, and R. Shankar, J. Phys. 6, 4909 (1994).

[4] H.-J. Kwon, A. Houghten, and J. B. Marston, Phys. Rev. Lett. 73, 284 (1994); Phys. Rev. B 52, 8002 (1995).

[5] A. H. Castro Neto and E. Fradkin, Phys. Rev. Lett. 72, 1393 (1994); Phys. Rev. B 49, 10877 (1994); Phys. Rev. B 51, 4048 (1995).

[6] J. Fröhlich, R. Götschmann, and P. A. Marchetti, J. Phys. A 28, 1169 (1995).

[7] P. Kopietz and K. Schönhammer, Z. Phys. B (in press).

[8] P. Kopietz, J. Hermisson, and K. Schönhammer, Phys. Rev. B 52, 10877 (1995).

[9] P. Kopietz, preprint cond-mat/9506132, to appear in the Proceedings of the Raymond L. Orbach Symposium, edited by D. Hone (World Scientific, 1995).

[10] P. Kopietz, Habilitationsschrift, Universität Göttingen (1995).

[11] C. Castellani, C. Di Castro and W. Metzner, Phys. Rev. Lett. 72, 316 (1994).

[12] C. Castellani and C. Di Castro, Physica C 235-240, 99 (1994).

[13] P. A. Lee, Phys. Rev. Lett. 63, 680 (1989); P. A. Lee amd N. Nagaosa, Phys. Rev. B 46, 5621 (1992); L. B. Ioffe and A. I. Larkin, Phys. Rev. B 39, 8988 (1989); B. Blok and H. Monien, Phys. Rev. B 47, 3454 (1993).

[14] B. I. Halperin, P. A. Lee, and N. Read, Phys. Rev. B 47, 7312 (1993).

[15] P. Kopietz, preprint cond-mat/9503090, to appear in Phys. Rev. B; J. Phys. A: Math. Gen. 28, L571 (1995).

[16] L. B. Ioffe, D. Lidsky, and B. L. Altshuler, Phys. Rev. Lett. 73, 472 (1994); B. L. Altshuler, L. B. Ioffe, and A. J. Millis, Phys. Rev. B 50, 14048 (1994).

[17] A. V. Svidzinskii, Zh. Eksp. Teor. Fiz. 31, 324 (1957) [Sov. Phys. JETP 4, 179 (1957)].

[18] B. M. Barbashov, Zh. Eksp. Teor. Fiz. 48, 607 (1965) [Sov. Phys. JETP 21, 402 (1965)].

[19] E. S. Fradkin, Nucl. Phys. 76, 588 (1966).

[20] V. N. Popov, Functional Integrals in Quantum Field Theory and Statistical Physics (Reidel, Dordrecht, 1983), chapter 4.

[21] D. V. Khveshchenko and P. C. E. Stamp, Phys. Rev. Lett. 71, 2118 (1993); Phys. Rev. B 49, 5227 (1994).

[22] We use the symbol $\mathcal{G}$ for the Green's function in a given fixed external field, and write $G=<\mathcal{G}>$ for the corresponding Green's function that is averaged with respect to an appropriate effective action.

[23] H. C. Fogedby, J. Phys. C 9, 3757 (1976); D. K. K. Lee and Y. Chen, J. Phys. A 21, 4155 (1988).

[24] Throughout this work we shall use the notation $k=\left[i \tilde{\omega}_{n}, \mathbf{k}\right], q=\left[i \omega_{m}, \mathbf{q}\right]$, and $\tilde{q}=$ $\left[i \tilde{\omega}_{n}, \mathbf{q}\right]$, where $\tilde{\omega}_{n}=2 \pi\left(n+\frac{1}{2}\right) T$ is a fermionic Matsubara frequency, and $\omega_{m}=2 \pi m T$ is a bosonic one. 
[25] R. Kubo, J. Phys. Soc. Japan 12, 570 (1957); P. C. Martin and J. Schwinger, Phys. Rev. 115, 1342 (1959).

[26] J. Schwinger, Phys. Rev. 128, 2425 (1962).

[27] S. Tomonaga, Prog. Theor. Phys. 5, 544 (1950); J. M. Luttinger, J. Math. Phys. 4, 1154 (1963); D. C. Mattis and E. H. Lieb, J. Math. Phys. 6, 304 (1965).

[28] J. Zinn-Justin, Quantum Field Theory and Critical Phenomena, (Clarendon Press, Oxford, 1989).

[29] See, for example, A. L. Fetter and J. D. Walecka, Quantum Theory of Many-Particle Systems, (McGraw-Hill, New York, 1991), p.158.

[30] I. E. Dzyaloshinskii and A. I. Larkin, Zh. Eksp. Teor. Fiz. 65, 411 (1973) [Sov. Phys. JETP 38, 202 (1974)]; A. Luther and I. Peschel, Phys. Rev. B 9, 2911 (1974).

[31] V. Meden and K. Schönhammer, Phys. Rev. B 46, 15753 (1992); K. Schönhammer and V. Meden, Phys. Rev. B 47, 16205 (1993); J. Voit, Phys. Rev. B 47, 6740 (1993).

[32] D. V. Khveshchenko, private communication.

[33] See, for example, D. Pines and P. Nozières, The Theory of Quantum Liquids, (AddisonWesley Advanced Book Classics, Redwood City, 1989).

[34] J. J. Quinn and R. A. Ferrell, Phys. Rev. 112, 812 (1958). 


\section{FIGURES}

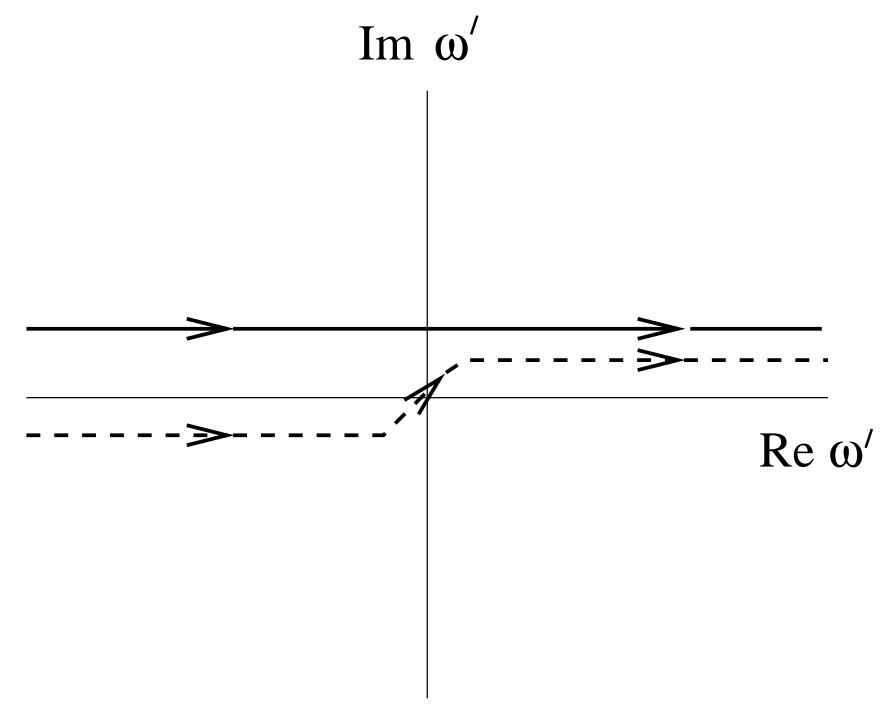

FIG. 1. Possible integration contours in the complex $\omega^{\prime}$-plane for the evaluation of Eq.42. The dashed line is the correct time-ordered contour. The solid line corresponds to choosing the retarded interaction in Eq.42. Both contours are infinitesimally close to the real axis. 